\title{
Otimização da desidratação osmótica de filés de mapará (Hypophthalmus edentatus) através da metodologia de superfície de resposta
}

\author{
Optimization of the osmotic dehydration of mapará (Hypophthalmus edentatus) \\ fillets by response surface methodology \\ Suezilde da Conceição Amaral RIBEIRO ${ }^{1}$, Kil Jin PARK², Miriam Dupas HUBINGER ${ }^{3}$, \\ Carmelita de Fátima Amaral RIBEIRO ${ }^{2 *}$, Eder Augusto Furtado ARAUJO ${ }^{4}$, Satoshi TOBINAGA ${ }^{3}$
}

\section{Resumo}

A desidratação osmótica de filés de mapará tem sido utilizada como pré-tratamento antes da secagem e refrigeração de alimentos como um meio de reduzir a umidade dos produtos. O objetivo do trabalho foi otimizar a transferência de massa ocorrida durante a desidratação osmótica de mapará através da metodologia de superfície de resposta. A desidratação osmótica foi realizada de acordo com um planejamento fatorial completo $2^{3}$, com oito pontos fatoriais, três centrais e seis axiais, totalizando 17 ensaios, em que o valor das variáveis dependentes, a perda de peso (PP), a perda de água (PA), o ganho de sólidos (GS), GS/PA e ácido tiobarbitúrico (TBA), são funções das variáveis independentes, temperatura, concentração de $\mathrm{NaCl}$ e tempo de imersão. A análise estatística aplicada aos dados experimentais foi realizada através do erro puro e pelo SS residual. Aplicando a metodologia de superfície de resposta, a condição ótima de convergência de menor perda de peso, maior perda de água e menor ganho de sal correspondeu a uma concentração de cloreto de sódio de $22 \%$, temperatura de $46{ }^{\circ} \mathrm{C}$ e tempo de 7 horas.

Palavras-chave: mapará; cloreto de sódio; concentração; temperatura; tempo de imersão.

\begin{abstract}
Osmotic dehydration of mapará fillets has been used as a pre-treatment before dehydration and refrigeration of food as a means of reducing product moisture content. The aim of this study was to optimize mass transfer occurring during osmotic dehydration of mapará using the response surface methodology. Osmotic dehydration was carried out following a $2^{3}$ complete factorial design, with eight factorial, three central and six axial points, totalizing 17 assays, where the values of the dependent variables, weight loss (PP), water loss (PA), solid gain (GS), GS/PA and tiobarbituric acid (TBA), are a function of the independent variables, temperature, concentration of $\mathrm{NaCl}$ and immersion time. The statistic analysis, applied to the experimental data was carried out using the pure error and the residual SS. Applying the response surface methodology, the best condition of convergence of lower weight loss, greater water loss and lower salt gain corresponded to a sodium chloride concentration of $22 \%$, temperature of $46^{\circ} \mathrm{C}$ and time of 7 hours.

Keywords: mapará; sodium chloride; concentration; temperature; immersion time.
\end{abstract}

\section{Introdução}

O mapará (Hypophthalmus edentatus) também conhecido como mapurá, peixe gato, mapará de cametá ou oleiro possui carne saborosa e alcança até $3 \mathrm{~kg}$ de peso, com um rendimento de filé superior a $60 \%$.

Segundo Alcântara-Neto (1994), a distribuição dos maparás é ampla na América do Sul; incluindo a Bacia Amazônica, a Bacia do Prata, de Orinoco e águas costeiras do Pará (foz do Amazonas) até o Suriname. Na Amazônia Central são encontradas as três espécies do gênero. O mapará é de interesse econômico, porém, da mesma forma que outros peixes lisos, o consumo no Amazonas é baixo, devido a tabus alimentares, sendo a maioria da produção comercializada para outros países e estados do Brasil.
A desidratação osmótica consiste na imersão do alimento, inteiro ou em pedaços, em soluções aquosas (por exemplo: sais, açúcar, sorbitol, glicerol, etc.) de alta pressão osmótica provocando a remoção da água presente no alimento (RAOULT- WACK et al., 1989; TORREGGIANI, 1993). Alimentos de origem animal como peixes, freqüentemente são processados em soluções aquosas, tendo o sal como o principal agente desidratante.

Esta imersão de alimentos, inteiros ou fatiados, em soluções hipertônicas, origina dois fluxos simultâneos e em contra corrente: um responsável pela saída da água do produto para a solução e o outro responsável pela migração de solutos da solução para o sólido. Um terceiro fluxo, também envolvido consiste na perda de alguns sólidos naturais, como açúcares,

Recebido para publicação em 18/6/2007

Aceito para publicação em 8/10/2007 (002611)

${ }_{1}$ Escola Agrotécnica Federal de Castanhal, Br 316, Km 62, CEP 68740970, Castanhal - PA, Brasil, E-mail: suzi@eafc-pa.gov.br

2 Faculdade de Engenharia Agrícola - FEAGRI, Unicamp, Cidade Universitária Zeferino Vaz, CP 6011, CEP 13083-875, Campinas - SP, Brasil,

E-mail: carmelita.ribeiro@agr.unicamp.br

3 Faculdade de Engenharia de Alimentos, Unicamp, E-mail: mhub@fea.unicamp.br

4 Departamento de Engenharia Química e de Alimentos, Universidade Federal do Pará - UFPA, E-mail: earaujo@ufpa.br

${ }^{*}$ A quem a correspondência deve ser enviada 
minerais, entre outros nutrientes que, embora sejam insignificantes proporcionalmente aos dois fluxos principais, podem ser importantes para a qualidade sensorial (aroma, cor e textura) e nutricional (minerais e vitaminas) do produto (RAOULTWACK et al., 1989).

A desidratação osmótica é um processo que geralmente envolve uma significante remoção de água (40 a 70 g de água é perdida por $100 \mathrm{~g}$ da massa inicial do produto) com uma limitada e controlada incorporação de sólidos (5 a 25 g de soluto ganho em $100 \mathrm{~g}$ da massa inicial) (RAOULT-WACK, 1994).

O objetivo deste trabalho foi otimizar a transferência de massa ocorrida durante a desidratação osmótica de filés de mapará mediante a metodologia de superfície de resposta.

\section{Material e métodos}

\subsection{Preparação das amostras}

Foi utilizado mapará (Hypophthalmus edentatus) proveniente da Edifrigo Comercial Industrial (Santarém-Pará). Os peixes foram lavados (com água clorada), eviscerados, embalados em sacos plásticos e congelados a $-18{ }^{\circ} \mathrm{C}$. Foram acondicionados em caixas de isopor com gelo e transportados para Campinas$\mathrm{SP}$, para fins de realização da presente pesquisa.

As amostras foram obtidas com o auxílio de um cortador de aço inox, desenvolvido para se obter placas de $3 \mathrm{~cm}$ de comprimento, $2 \mathrm{~cm}$ de largura e $0,5 \mathrm{~cm}$ de espessura.

\subsection{Análise microbiológica da matéria-prima}

Foi realizada a análise microbiológica quanto à detecção de Salmonella e Staphylococcus aureus do peixe de acordo com a Resolução - RDC no 12, de 02 de janeiro de 2001, da Agência Nacional de Vigilância Sanitária - ANVISA. Como a matériaprima foi manipulada antes de chegar a Campinas, foi realizada a análise de Coliformes fecais de acordo com a Portaria 451 de 19/09/1997 do Ministério da Saúde, para verificar a sanidade do lote recebido.

\subsection{Análise físico-química da matéria-prima}

Foi realizada a caracterização físico-química da matériaprima através de determinações de umidade, cinzas, proteínas e lipídios do mapará, utilizando a metodologia da AOAC (1995). A umidade foi determinada em estufa a $105^{\circ} \mathrm{C}$ até peso constante. Os resíduos minerais foram determinados por incineração da matéria em mufla a $550{ }^{\circ} \mathrm{C}$, até peso constante. A proteína foi determinada mediante a determinação do nitrogênio total, pelo método Kjeldahl (6,25 como fator de correção) e lipídios determinado pelo método de Bligh e Dyer (1959), utilizando clorofórmio e metanol como solvente.

\subsection{Processo de desidratação osmótica}

As amostras devidamente cortadas na geometria de placa plana foram pesadas e colocadas em béquer de vidro de $600 \mathrm{~mL}$, contendo solução de $\mathrm{NaCl}$ aquecida à temperatura de processo. A relação amostra/solução de 1:5 foi utilizada para garantir a concentração constante da solução osmótica. O conjunto amostra-solução desidratante foi levado à incubadora refrigerada "shaker" da marca TECNAL (modelo TE - 421) e mantido na temperatura de estudo. Foi adotada uma agitação constante de 80 rpm, pois esta agitação provia uma movimentação satisfatória da amostra dentro da solução, sem que a mesma sofresse impacto com as paredes do béquer.

As amostras, depois de um tempo pré-estabelecido pelo planejamento experimental, foram retiradas do "shaker", lavadas com água destilada para retirar o excesso da solução, depois envolvidas em papel absorvente para remoção do excesso de água, e foram então novamente pesadas.

A perda de peso, a perda de água e o ganho de sólidos dos filés de mapará submetidos a tratamento osmótico foram calculados pelas seguintes equações (Equação 1, 2 e 3):

Perda de peso (PP)

$$
\mathrm{PP}(\%)=\frac{\left(\mathrm{MA}_{0}-\mathrm{M}_{\mathrm{t}}\right)}{\mathrm{MA}_{0}} \times 100
$$

Perda de água (PA)

$\mathrm{PA}(\%)=\frac{\mathrm{MA}_{0}-\left(\mathrm{M}_{\mathrm{t}}-\mathrm{MS}_{\mathrm{t}}\right)}{\mathrm{MA}_{0}+\mathrm{MS}_{0}} \times 100$

Ganho de sólidos (SG)

$$
\mathrm{SG}(\%)=\frac{\mathrm{MS}_{\mathrm{t}}-\mathrm{MS}_{0}}{\mathrm{MA}_{0}+\mathrm{MS}_{0}} \times 100
$$

onde:

$\mathrm{MA}_{0}=$ massa da água inicialmente presente na amostra (g);

$\mathrm{MS}_{0}=$ massa dos sólidos inicialmente presentes na amostra (g);

$\mathrm{M}_{\mathrm{t}}=$ massa da amostra no tempo $\mathrm{t}(\mathrm{g})$; e

$\mathrm{MS}_{\mathrm{t}}=$ massa dos sólidos no tempo $\mathrm{t}(\mathrm{g})$.

\subsection{Número de ácido tiobarbitúrico (TBA)}

Após a desidratação osmótica foi determinado o número de ácido tiobarbitúrico (TBA) de acordo com a metodologia de Tarladgis et al. (1960). Os destilados obtidos foram submetidos à reação de cor com ácido 2-tiobarbitúrico e lidos em espectrofotômetro a $532 \mathrm{~nm}$.

\subsection{Tratamento estatístico dos dados}

Foi utilizado um planejamento fatorial completo $2^{3}$, com pontos centrais (nível 0) e pontos axiais (níveis $\pm \alpha$ ). Os pontos axiais $( \pm \alpha)$ são utilizados para a ampliação do modelo linear, tornando-o quadrático. $\mathrm{O}$ valor de $\alpha$ é função do número de variáveis independentes (k), sendo definido pela Equação 4 (BARROS NETO et al., 2001).

$\alpha=\left(2^{k}\right)^{1 / 4}$ 
Como são três variáveis independentes, o valor de $\alpha$ é 1,6818 .

Na Tabela 3 estão apresentados os níveis reais e codificados dos fatores estudados. Foram realizados 17 ensaios, sendo oito fatoriais (combinação entre os níveis \pm 1 ), três centrais (três variáveis no nível 0 ) e seis axiais (uma variável no nível $\pm \alpha$ e duas no nível 0 ), gerando um modelo quadrático cujo valor das variáveis dependentes (perda de peso (PP), perda de água (PA), ganho de sólidos (GS), GS/PA e TBA) é função das variáveis independentes, temperatura $(\mathrm{T})$, concentração $(\mathrm{C})$ e tempo $(\mathrm{t})$, conforme descreve a Equação 5 abaixo:

$Y=f(T, C, t)=\beta_{0}+\beta_{1} T+\beta_{11} T^{2}+\beta_{2} C+\beta_{22} C^{2}+$

$\beta_{3} \mathrm{t}+\beta_{33} \mathrm{t}^{2}+\beta_{12} \mathrm{TC}+\beta_{13} \mathrm{Tt}+\beta_{23} \mathrm{Ct}$

Como nem sempre os coeficientes mostrados na Equação 5 são estatisticamente significativos, foi necessário realizar uma análise estatística apropriada para assegurar a validade destes coeficientes dentro de um nível de confiança estabelecido (p).

Após a análise estatística dos coeficientes, a influência dos fatores e suas interações sobre as respostas foram analisadas através de análise de variância (ANOVA), teste F e coeficiente de determinação $\left(\mathrm{R}^{2}\right)$, verificando desta maneira se o modelo representa um grau de ajuste adequado aos dados experimentais.

A otimização do processo osmótico dos filés de mapará teve como objetivo manter as características iniciais do pescado in natura, utilizando temperatura, concentração da solução e tempo de desidratação que não provocassem alterações significativas na estrutura do material.

A melhor condição de processamento de desidratação osmótica foi determinada utilizando Metodologia de Superfície de Resposta (RSM), realizando a sobreposição dos gráficos de GS/PA e TBA para maximizar a perda de água e minimizar o ganho de sólidos e TBA.

\section{Resultados e discussão}

\subsection{Análise microbiológica da matéria-prima}

Os resultados da análise microbiológica da matéria-prima estão apresentados na Tabela 1 . Foi comprovada que a matériaprima congelada estava dentro dos limites da legislação brasileira, portanto, apta para o processamento e/ou consumo.

A ausência de coliformes fecais, Staphylococcus aureus e Salmonella confirma que os procedimentos sanitários e higiênicos foram corretamente seguidos desde a captura até a preparação da matéria-prima. Se houvesse a confirmação da presença de tais bactérias, a matéria-prima deveria ser descartada para impedir qualquer tipo de contaminação.

Tabela 1. Caracterização microbiológica da matéria-prima.

\begin{tabular}{lcc}
\hline \multicolumn{1}{c}{ Análises } & Mapará in natura & Legislação \\
\hline $\begin{array}{l}\text { Coliformes fecais } \\
\text { Salmonella }\end{array}$ & AMP $<3 / \mathrm{g}^{*}$ & Max $10^{2} / \mathrm{g}$ \\
Staphylococcus aureus $25 \mathrm{~g}$ & $<1 \times 10^{2}$ u.f.c $/ \mathrm{g}^{* *}$ & Ausência em $25 \mathrm{~g}$ \\
$\begin{array}{l}\text { NMP: número mais provável; e } \\
\text { amostra. }\end{array}$ &
\end{tabular}

Alimentos que sofrem manipulação são potencialmente capazes de causar intoxicação estafilocócica e os manipuladores são importantes fontes de contaminação de S. aureus (NETO et al., 2002). Trabalhos realizados por Andrade e Zelante (1989) avaliando a ocorrência de S. aureus enterotoxigênicos nas mãos, na boca e nas fezes, em portadores assintomáticos, verificaram que $24,8 \%$ das linhagens isoladas foram enterotoxigênicas. Resultados similares foram descritos por Pereira et al. (1994), que trabalhando com manipuladores de alimentos de cozinha industrial encontraram 30,9\% de cepas enterotoxigênicas dos 55 isolados de $S$. aureus.

Segundo Franco e Guth (1985), além da gravidade da constatação do alimento estar contaminado por microrganismos de origem fecal, a presença de E. coli pode ter um significado particularmente importante, uma vez que se constitui em linhagens enteropatogênicas, causadoras de vários surtos e gastroenterites, provocados pela ingestão de água e/ou alimentos contaminados.

A Salmonella constitui um alto risco à saúde do consumidor por ser um contaminante de alimentos manipulados sem cuidados higiênico-sanitários, além do armazenamento e transporte dos mesmos serem feitos de maneira inadequada. Alimentos in natura de origem animal são as maiores fontes de Salmonella, e o risco de se contrair uma salmonelose tem início quando esses alimentos, já contaminados, entram em contato direto com o manipulador, que poderá servir como agente disseminador desses microrganismos aos equipamentos, utensílios e alimentos já preparados (ROITMAN et al., 1988).

\subsection{Análise físico-química da matéria-prima}

Os resultados da análise físico-química de umidade, cinzas, proteínas e lipídios estão apresentados na Tabela 2.

Comparando os dados aos encontrados por Oliveira e Soares (1999) que, realizando a composição química do mapará in natura, determinaram que este continha $67,7 \%$ de umidade, $12,5 \%$ de proteína, $18,3 \%$ de lipídios e $0,7 \%$ de cinzas, verificase que apenas a porcentagem de lipídios foi compatível, porém os outros valores estão relativamente próximos. Os valores de cinzas e proteínas são muito parecidos aos encontrados por Lourenço et al. (1999), que estudaram as características químicas do mapará in natura e também salgado e seco e verificaram que a composição química do músculo in natura apresentava $80,83 \%$ de umidade, $17,18 \%$ de proteína, $1,16 \%$ de lipídios e $0,83 \%$ de cinzas, e o músculo salgado e seco apresentou $33,87 \%$ de umidade, 33,45\% de proteína, 9,60\% de lipídios, 23,08\% de cinzas e $18,20 \%$ de $\mathrm{NaCl}$.

Segundo Dean (1990), o pescado pode conter de 60 a $65 \%$ de umidade e 0,6 a $36 \%$ de lipídios, sendo que este último

Tabela 2. Caracterização físico-química da matéria-prima.

\begin{tabular}{ccccc}
\hline $\begin{array}{c}\text { Componentes } \\
\text { (base úmida) }(\%)\end{array}$ & $\begin{array}{c}\text { Valor } \\
\text { mínimo }\end{array}$ & $\begin{array}{c}\text { Valor } \\
\text { máximo }\end{array}$ & $\begin{array}{c}\text { Média dos } \\
\text { valores }\end{array}$ & $\begin{array}{c}\text { Desvio } \\
\text { padrão }\end{array}$ \\
\hline Umidade & 63,98 & 64,03 & 64,01 & 0,03 \\
Lipídios & 17,67 & 18,01 & 17,85 & 0,17 \\
Proteínas & 16,29 & 16,43 & 16,35 & 0,07 \\
Cinzas & 0,88 & 0,93 & 0,91 & 0,03 \\
\hline
\end{tabular}


componente apresenta esta grande variação em vista do tipo de músculo corporal analisado (a carne dorsal apresenta menor quantidade lipídica que a carne abdominal), espécie de peixe, sexo, idade, época do ano, habitat e dieta.

Os peixes gordurosos apresentam grande variação no teor de água com a época do ano. De um modo geral, conforme aumenta o teor de gordura no músculo do pescado, diminui o teor de água na mesma proporção. Desta forma, o teor de água no músculo de pescado gordo situa-se, de um modo geral, na faixa de 60 a 75\%, dependendo do pescado e da época do ano (GEROMEL; FORSTER, 1982), como no caso do mapará utilizado neste trabalho.

Contreras-Guzmán (1994) relata que a fração de cinzas em peixes de água doce apresenta variações em quantidades que vão desde 0,90 a 3,39\%. Este intervalo é compatível ao valor de cinzas encontrado (Tabela 2 ). Com relação aos minerais, a carne de pescado é considerada uma fonte valiosa de cálcio e fósforo, particularmente, apresentando também quantidades razoáveis de sódio, potássio, manganês, cobre, cobalto, zinco, ferro e iodo.

\subsection{Desidratação osmótica de filés de mapará}

$\mathrm{Na}$ Tabela 3 estão apresentados os valores de perda de peso, perda de água, ganho de sólidos, TBA e a razão GS/PA, obtidos experimentalmente do processo de desidratação osmótica de filés de mapará, seguindo a planilha do planejamento experimental completo $2^{3}$.

\section{Efeitos das variáveis sobre as respostas}

Os resultados da análise estatística, aplicados aos dados experimentais de perda de peso, perda de água, ganho de sólidos e TBA foram determinados através do erro puro e pelo $\mathrm{SS}$ residual.
$\mathrm{O}$ efeito estimado indica o quanto cada fator influi nas respostas estudadas (neste caso, PP, PA, GS e TBA). Quanto maior é o seu valor, maior é a sua influência, e um efeito positivo indica que ao passar de um valor mínimo a um valor máximo da variável, a resposta aumenta. Já um efeito negativo indica o contrário, ou seja, ao passar de um valor mínimo para o valor máximo, a resposta diminui.

Na Figura 1 estão apresentados gráficos de barras dos valores dos efeitos estimados da temperatura, concentração e tempo sobre as respostas perda de peso, perda de água, ganho de sólidos e TBA durante a desidratação osmótica de filés de mapará.

Os fatores significativos foram os mesmos para a perda de peso, tanto levando em consideração o erro puro como o SS residual. Verifica-se que os parâmetros lineares de temperatura, concentração e tempo apresentam efeito positivo para perda de peso, ou seja, um aumento em qualquer um desses fatores acarreta um aumento na perda de peso. Verifica-se também que a temperatura linear é a que apresenta maior efeito sobre a perda de peso.

Na perda de água os parâmetros lineares significativos de temperatura, concentração e tempo apresentam um efeito positivo, ou seja, um aumento em qualquer um desses fatores acarreta um aumento na perda de água. Observa-se também que o parâmetro temperatura linear é o que apresenta maior efeito.

Os parâmetros de concentração linear e concentração quadrática apresentam um efeito positivo no ganho de sólidos, ou seja, um aumento em qualquer um desses fatores acarreta um aumento no ganho de sólidos, enquanto os parâmetros temperatura linear e a interação temperatura $\times$ tempo apresentam um efeito negativo, ou seja, um aumento nestes fatores acarreta na diminuição do ganho de sólidos. Observa-se ainda, que o parâmetro temperatura linear é o que apresenta maior efeito no ganho de sólidos.

Tabela 3. Perda de peso, perda de água, ganho de sólidos, TBA e GS/PA obtidos experimentalmente durante a desidratação osmótica de filés de mapará utilizando solução binária de $\mathrm{NaCl}$.

\begin{tabular}{|c|c|c|c|c|c|c|c|c|c|c|c|}
\hline & \multicolumn{3}{|c|}{ Codificado } & \multicolumn{3}{|c|}{ Real } & \multicolumn{5}{|c|}{ Respostas } \\
\hline & $\mathrm{T}\left({ }^{\circ} \mathrm{C}\right)$ & C (\%) & $\mathrm{t}(\mathrm{h})$ & $\mathrm{T}\left({ }^{\circ} \mathrm{C}\right)$ & $\begin{array}{l}\text { Conc. de } \\
\mathrm{NaCl}(\%)\end{array}$ & $\mathrm{t}(\mathrm{h})$ & PP (\%) & PA (\%) & GS (\%) & $\begin{array}{c}\text { TBA } \\
\text { (mg Mal./1000 g) }\end{array}$ & GS/PA \\
\hline 1 & -1 & -1 & -1 & 30 & 21 & 6 & 4,401 & 15,402 & 11,001 & 0,041 & 0,714 \\
\hline 2 & +1 & -1 & -1 & 50 & 21 & 6 & 19,657 & 26,707 & 7,050 & 0,302 & 0,264 \\
\hline 3 & -1 & +1 & -1 & 30 & 25 & 6 & 5,072 & 21,893 & 16,821 & 0,310 & 0,768 \\
\hline 4 & +1 & +1 & -1 & 50 & 25 & 6 & 23,251 & 32,321 & 9,070 & 0,982 & 0,281 \\
\hline 5 & -1 & -1 & +1 & 30 & 21 & 12 & 6,431 & 20,930 & 14,499 & 0,705 & 0,693 \\
\hline 6 & +1 & -1 & +1 & 50 & 21 & 12 & 24,230 & 32,695 & 8,465 & 0,824 & 0,259 \\
\hline 7 & -1 & +1 & +1 & 30 & 25 & 12 & 5,686 & 25,449 & 19,763 & 1,026 & 0,777 \\
\hline 8 & +1 & +1 & +1 & 50 & 25 & 12 & 26,570 & 35,023 & 8,453 & 1,658 & 0,241 \\
\hline 9 & $-\alpha$ & 0 & 0 & 23 & 23 & 9 & 1,516 & 14,998 & 13,482 & 0,400 & 0,899 \\
\hline 10 & $+\alpha$ & 0 & 0 & 57 & 23 & 9 & 30,024 & 36,496 & 6,471 & 1,235 & 0,177 \\
\hline 11 & 0 & $-\alpha$ & 0 & 40 & 20 & 9 & 9,346 & 22,033 & 12,687 & 0,077 & 0,576 \\
\hline 12 & 0 & $+\alpha$ & 0 & 40 & 26 & 9 & 14,072 & 30,341 & 16,269 & 0,722 & 0,536 \\
\hline 13 & 0 & 0 & $-\alpha$ & 40 & 23 & 4 & 10,858 & 23,401 & 12,543 & 0,206 & 0,536 \\
\hline 14 & 0 & 0 & $+\alpha$ & 40 & 23 & 14 & 20,221 & 31,866 & 11,645 & 1,150 & 0,365 \\
\hline 15 & 0 & 0 & 0 & 40 & 23 & 9 & 16,579 & 27,773 & 11,194 & 0,442 & 0,403 \\
\hline 16 & 0 & 0 & 0 & 40 & 23 & 9 & 15,134 & 26,208 & 11,074 & 0,462 & 0,423 \\
\hline 17 & 0 & 0 & 0 & 40 & 23 & 9 & 15,861 & 26,991 & 11,130 & 0,418 & 0,412 \\
\hline
\end{tabular}




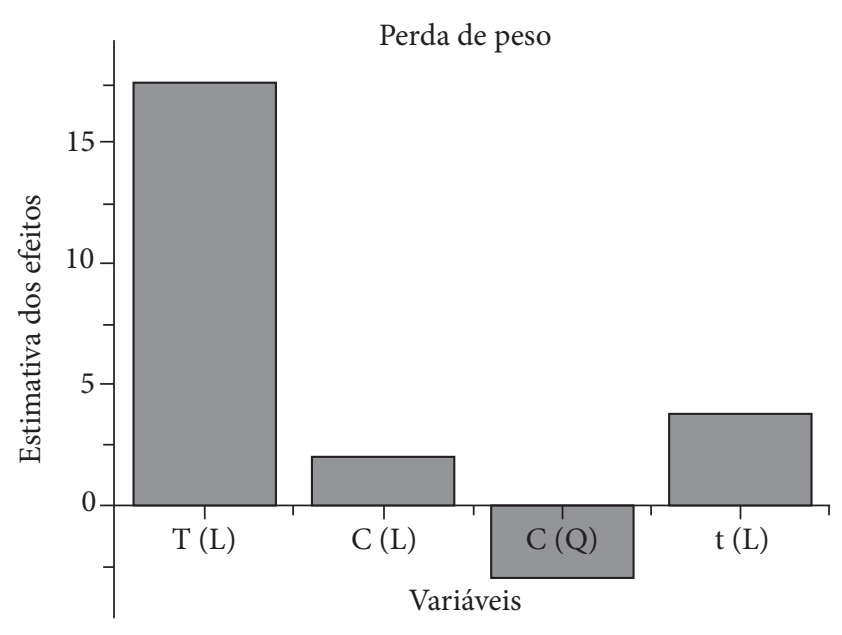

Ganho de sólidos

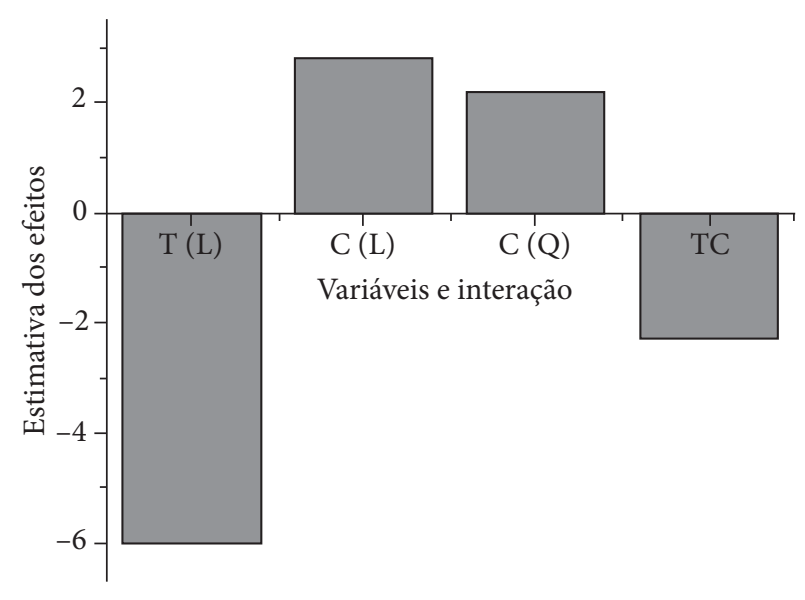

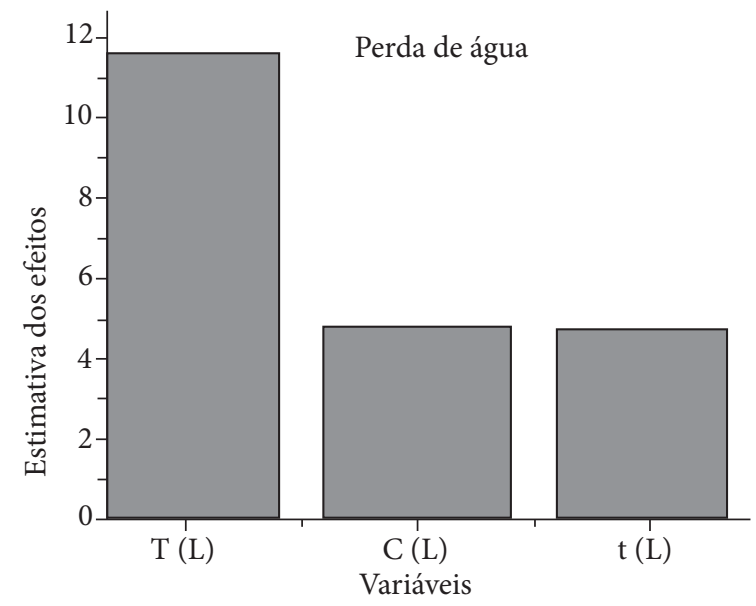

TBA (ácido tiobarbitúrco)

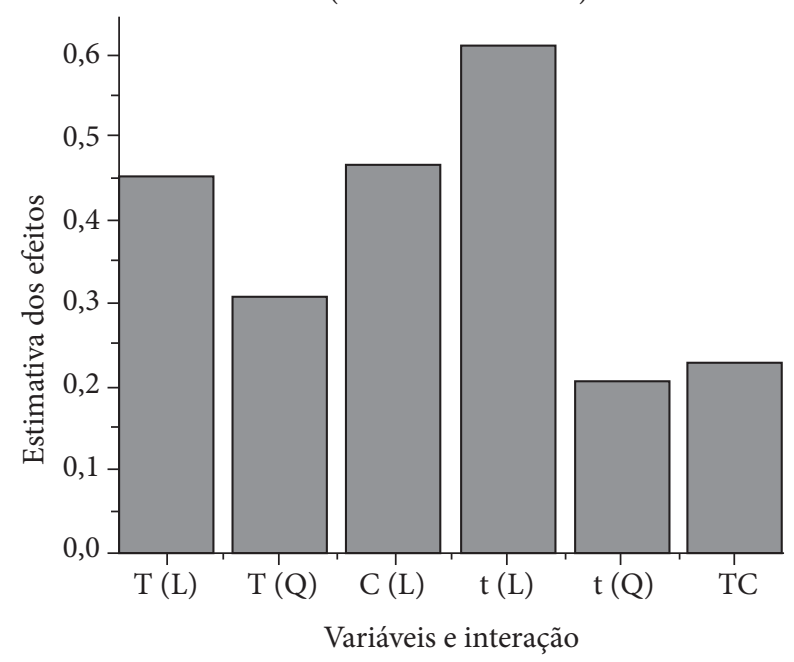

Figura 1. Efeitos estimados para a perda de peso, perda de água, ganho de sólidos e TBA durante a desidratação osmótica de filés de mapará.

Na determinação do número de ácido tiobarbitúrico o fator que tem mais efeito sobre a resposta é o tempo linear, seguido da concentração linear, temperatura linear, temperatura quadrática, da interação temperatura $x$ concentração e do tempo quadrático. Todos os fatores significativos e interação têm efeito positivo na resposta, ou seja, com o aumento de qualquer um destes, há o aumento do número de ácido tiobarbitúrico (TBA).

Após a eliminação dos parâmetros com efeitos não significativos de perda de peso, perda de água, ganho de sólidos e TBA, verificou-se através da análise de variância (ANOVA) a significância da regressão e da falta de ajuste com $95 \%$ de confiança $(\mathrm{p} \leq 0,05)$, utilizando o teste $F$ para o planejamento estudado, conforme a Tabela 4.

A análise de variância (Tabela 4) mostrou que o modelo ajustado para a perda de peso (Equação 6) foi significativo com 95\% de confiança e também preditivo, satisfazendo o critério sugerido por Box e Wetz (1973), visto que o F calculado foi maior que o $\mathrm{F}$ tabelado e a falta de ajuste não foi significativa no mesmo nível de confiança. O coeficiente de determinação $\left(\mathrm{R}^{2}\right)$ foi de 0,98 , indicando que o modelo explicou $98 \%$ da variação dos dados observados.
O coeficiente de determinação $\left(\mathrm{R}^{2}\right)$ foi de 0,98 , indicando que o modelo explicou $98 \%$ da variação dos dados observados (Equação 7). O modelo apresentou regressão significativa com 95\% de confiança (F calculado superior ao F tabelado) e falta de ajuste não significativo no mesmo nível de confiança (F calculado inferior ao F tabelado). Sendo assim, o modelo ajustado para perda de água no processo de desidratação osmótica foi considerado preditivo.

Analisando os valores obtidos de ganho de sólidos na Tabela 4, observa-se que o modelo apresentou regressão significativa (Equação 8), mas o modelo não pode ser considerado preditivo, pois a falta de ajuste também foi significativa ( $F$ calculado superior ao $\mathrm{F}$ tabelado). $\mathrm{O}$ alto valor de $\mathrm{F}$ calculado para a falta de ajuste é devido ao fato de que o mínimo quadrado (MQ) do erro puro apresenta um valor muito inferior ao mínimo quadrado da falta de ajuste (devido à boa repetição dos ensaios dos pontos centrais), isso faz com que a razão entre eles resulte num valor muito alto. $\mathrm{O}$ coeficiente de determinação $\left(\mathrm{R}^{2}\right)$ obtido foi de 0,87 , indicando que o modelo explicou $87 \%$ da variação dos dados experimentais. 
Tabela 4. Análise de variância (ANOVA) para a perda de peso, perda de água, ganho de sólidos e TBA durante a desidratação osmótica de filés de mapará.

\begin{tabular}{|c|c|c|c|c|c|c|}
\hline \multicolumn{7}{|c|}{ Perda de peso } \\
\hline Fonte de Variação & SQ & GL & MQ & $\mathrm{F}_{\text {calculado }}$ & $\mathrm{F}_{\text {tabelado }}(\mathrm{p} \leq 0,05)$ & $\mathrm{R}^{2}$ \\
\hline Regressão & 1146,12 & 4 & 286,53 & 174,71 & 3,26 & 0,98 \\
\hline Resíduo & 19,64 & 12 & 1,64 & - & - & - \\
\hline Erro puro & 1,05 & 2 & 0,52 & - & - & - \\
\hline Total & 1165,76 & 16 & - & - & - & - \\
\hline Resíduo & 14,71 & 13 & 1,13 & - & - & - \\
\hline Falta de ajuste & 13,49 & 11 & 1,23 & 2,00 & 19,40 & \\
\hline Erro puro & 1,22 & 2 & 0,61 & - & - & - \\
\hline Total & 628,74 & 16 & - & - & - & - \\
\hline \multicolumn{7}{|c|}{ Ganho se sólidos } \\
\hline Total & 202,71 & 16 & - & - & - & - \\
\hline \multicolumn{7}{|c|}{ TBA (nvúmero de ácido tiobarbitúrico) } \\
\hline Regressão & 3,16 & 6 & 0,63 & 98,81 & 3,22 & 0,98 \\
\hline Resíduo & 0,06 & 10 & 0,01 & - & - & - \\
\hline Falta de ajuste & 0,06 & 8 & 0,01 & 15,60 & 19,37 & - \\
\hline Erro puro & 0,00 & 2 & 0,00 & - & - & - \\
\hline Total & 3,23 & 16 & - & - & - & - \\
\hline
\end{tabular}

O modelo codificado para TBA (Equação 9) apresentou regressão significativa a $95 \%$ de confiança. O valor da razão $\mathrm{MQ}_{\mathrm{R}} / \mathrm{MQ}_{\mathrm{r}}$ é 98,81. Comparado com $\mathrm{F}=3,22$ este valor indica uma regressão significativa e também útil para fins preditivos.

Segundo Barros Neto et al. (2001), uma regressão, embora significativa do ponto de vista do teste $F$, pode não ser útil para realizar previsões por cobrir uma faixa de variação pequena dos fatores estudados. Box e Wetz (1973) sugeriram que para uma regressão ser significativa não apenas estatisticamente, mas também ser útil para fins preditivos, o valor de F calculado para a regressão deve ser no mínimo quatro a cinco vezes maior que o valor de F tabelado. Já o F da falta de ajuste em relação ao erro puro, pelo contrário, deve apresentar o menor valor possível, pois um alto $\mathrm{F}$ indica que há uma grande falta de ajuste dos dados ao modelo obtido.

\section{Obtenção dos modelos}

Os modelos codificados propostos para representar perda de peso (PP), perda de água (PA), ganho de sólidos (GS) e número de ácido tiobarbitúrico (TBA) foram ajustados através de regressão em função dos fatores temperatura (T), concentração de $\mathrm{NaCl}(\mathrm{C})$ e tempo (t), conforme os seguintes modelos:

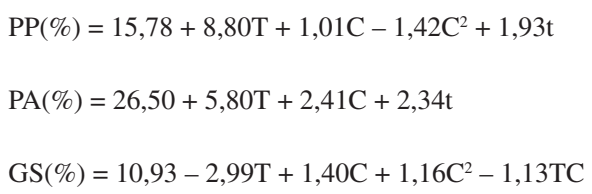

TBA $\left(\frac{\text { mg malon. }}{1000 \mathrm{~g}}\right)=0,44+0,23+0,15 \mathrm{~T}^{2}+$
$0,23 \mathrm{C}+0,31 \mathrm{t}+0,10 \mathrm{t}^{2}+0,12 \mathrm{TC}$

\subsection{Otimização dos níveis e fatores sobre as respostas na desidratação osmótica de mapará}

Nas Figuras 2, 3 e 4, o gráfico de GS/PA está representado através das áreas, enquanto que o gráfico de TBA está delimitado através de linhas. Observa-se uma região branca, considerada ótima para o estudo, pois é uma região limite do objetivo de obter produtos com maior perda de água, menor ganho de sólidos e menor oxidação, ou seja, baixo valor de TBA, sem modificar drasticamente as características iniciais do pescado.

Dentro da faixa de trabalho escolhida, que varia de 0,258 a 0,336 para GS/PA e 0,397 a 0,653 para TBA, das Figuras 2, 3 e 4, levando-se em consideração que uma maior temperatura e uma maior concentração de $\mathrm{NaCl}$ poderiam provocar uma maior desnaturação de proteínas alterando a textura e também um escurecimento por oxidação de lipídios, afetando a cor, o sabor e o odor do mapará, opta-se pela seguinte condição ótima: temperatura de $46{ }^{\circ} \mathrm{C}$, concentração de $22 \%$ e tempo de 7 horas. A textura da carne também pode ser alterada pela oxidação devido à formação de complexos entre a proteína do pescado e os produtos de oxidação, provocando o enrijecimento da carne (KHAYAT; SCHWALL, 1983; KANNER, 1994; BAK et al., 1999). 


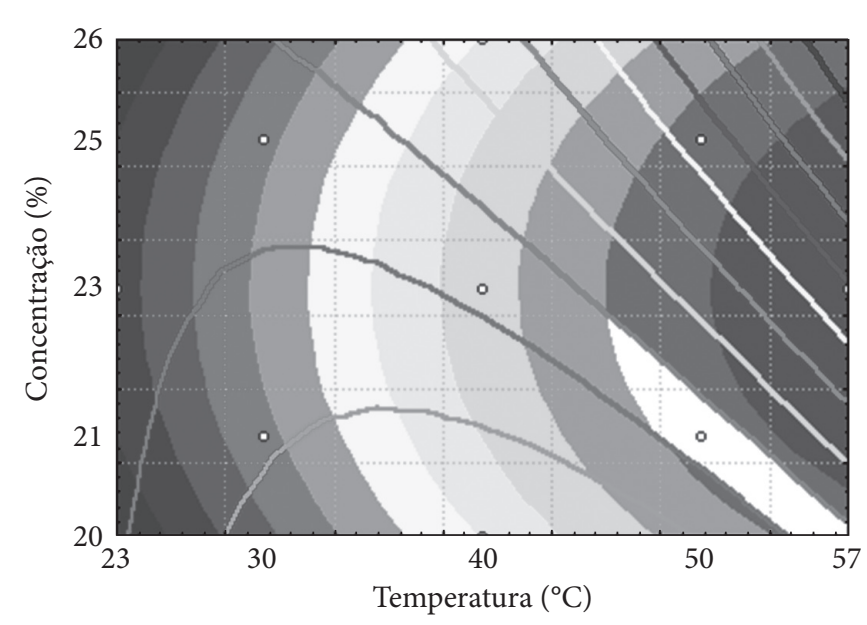

\begin{tabular}{|llll|}
$\square 0,227$ & $\square 0,469$ & $\square 0,712$ & 0,955 \\
$\square 0,308$ & $\square 0,550$ & $\square 0,793$ & above \\
$\square 0,389$ & $\square 0,631$ & $\square 0,874$ & \\
$-0,223$ & $-0,746$ & $-1,270$ & $-1,793$ \\
$-0,397$ & $-0,921$ & $-1,444$ & \\
$-0,572$ & $-1,095$ & $-1,618$ & \\
\hline
\end{tabular}

Figura 2. Sobreposição das curvas de contorno (GS/PA) e TBA no tempo de 9 horas durante a desidratação osmótica de filés de mapará em solução de $\mathrm{NaCl}$.

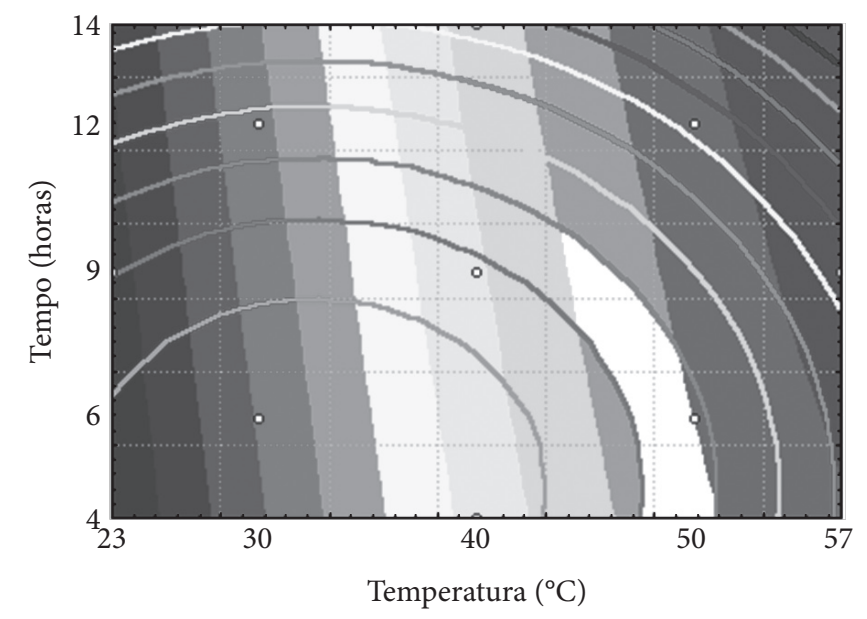

\begin{tabular}{|llll|}
$\square 1,181$ & $\square 0,413$ & $\square 0,646$ & $\square 0,879$ \\
$\square 0,258$ & $\square 0,491$ & $\square 0,724$ & $\square$ above \\
$\square 0,336$ & $\square 0,568$ & $\square 0,801$ & \\
$-0,304$ & $-0,828$ & $-1,352$ & $-1,876$ \\
$-0,479$ & $-1,002$ & $-1,526$ & \\
$-0,653$ & $-1,177$ & $-1,701$ & \\
\hline
\end{tabular}

Figura 3. Sobreposição das curvas de contorno (GS/PA) e TBA na concentração de $23 \%$ durante a desidratação osmótica de filés de mapará em solução de $\mathrm{NaCl}$.

\section{Conclusões}

O mapará apresentou altos teores de lipídios e proteínas, sendo assim caracterizado como um peixe gordo e de potencial nutritivo, portanto ótimo para a aplicação de tecnologias e ob-

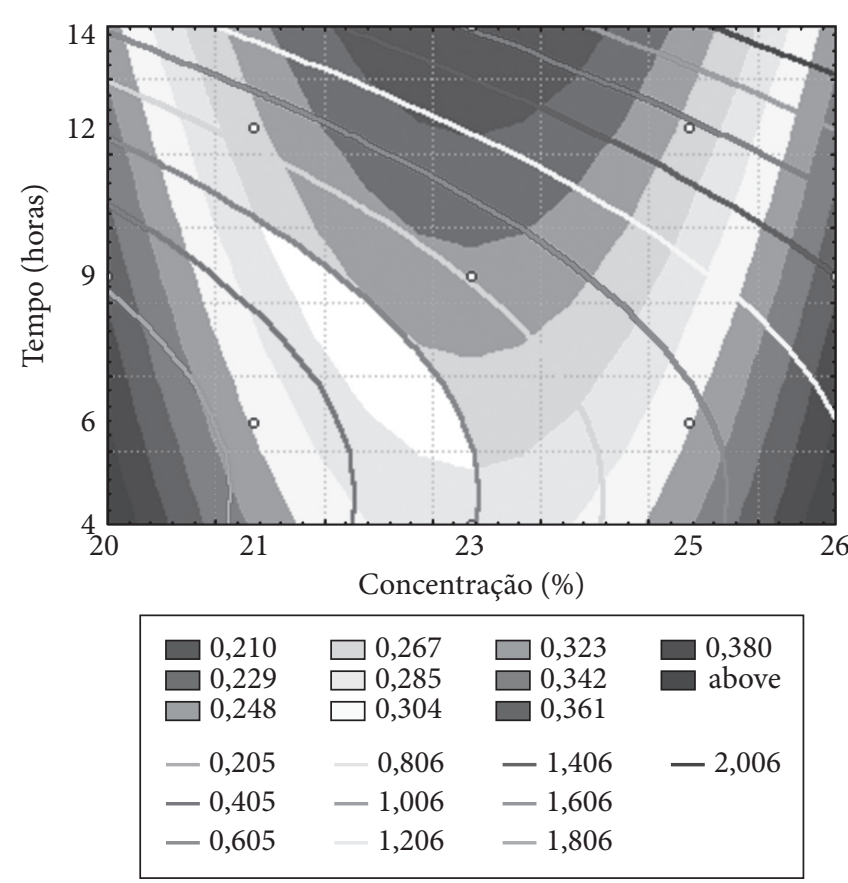

Figura 4. Sobreposição das curvas de contorno (GS/PA) e TBA na temperatura de $50{ }^{\circ} \mathrm{C}$ durante a desidratação osmótica de filés de mapará em solução de $\mathrm{NaCl}$.

tenção de novos produtos de valor nutricional. Os resultados das análises microbiológicas comprovaram que os peixes estavam dentro dos limites exigidos pela Legislação Brasileira.

Através da otimização das respostas na desidratação osmótica de filés de mapará foi possível modelar as respostas de perda de peso, perda de água, ganho de sólidos e TBA que ocorrem durante o processo de desidratação osmótica, podendo ser aplicadas satisfatoriamente para restringir critérios econômicos e de estabilidade do produto.

Os modelos codificados para representar perda de peso $(\mathrm{PP})$, perda de água $(\mathrm{PA})$ e número de ácido tiobarbitúrico (TBA) em função dos fatores temperatura (T), concentração de $\mathrm{NaCl}(\mathrm{C})$ e tempo (t) foram preditivos.

A condição ótima de convergência de menor perda de peso, maior perda de água e menor ganho de sal foi a uma concentração de cloreto de sódio de $22 \%$ de $\mathrm{NaCl}$, temperatura de $46^{\circ} \mathrm{C}$ e tempo de 7 horas.

\section{Referências bibliográficas}

ALCÂNTARA NETO, C. P. Ecologia da pesca dos maparás, Hypophthalmus spp. (Siluriformes, Hypophthalmidae), no lago Grande de Monte Alegre, Baixo Amazonas, Pará. Belém, 1994. 141 p. Dissertação, Universidade Federal do Pará/Museu Paraense Emílio Goeldi.

ANDRADE, G. P.; ZELANTE, F. Ocorrência simultânea de Staphylococcus aureus enterotoxigênicos nas mãos, boca e fezes em portadores assintomáticos. Revista de Saúde pública, São Paulo, v. 23, n. 4, p. 277-84, 1989.

ANVISA - Agência Nacional de Vigilância Sanitária. Resolução RDC n. 12, de 02 de janeiro de 2001. Regulamento Técnico Sobre 
Padrões Microbiológicos Para Alimentos. Disponível em:< http://e-legis.anvisa.gov.br/leisref/public/showAct.php?id=144>.

AOAC - Association Official Analytical Chemists. Official Methods of Analysis. 14 ed. Arlington, 1995.

BAK, L. S. et al. Effect of modified atmosphere packaging on oxidative chages in frozen cold water shrimp (Pandalus borealis). Food Chemistry, Reading, v. 64, n. 2, p. 169-175, 1999.

BARROS NETO, B.; SCARMINIO, I. S.; BRUNS, R. E. Como fazer experimentos-pesquisa e desenvolvimento na ciência e na indústria. Campinas: Editora da UNICAMP, 2001.

BLIGH, E. G.; DYER, W. J. A rapid method of total lipid extration and purification. Canadian Journal Biochemistry Physiology, Ottawa, v. 37, n. 8, p. 911-917, 1959.

BOX, G. E. P.; WETZ, J. Criteria for judging adequacy of estimation by an approximate response function. University of Wisconsin Technical Report, Madison, n. 9, 1973.

CONTRERAS-GUZMÁN, E. S. Bioquímica de pescados e derivados. Jabuticabal: Funep, 1994. 409 p.

DEAN, L. M. Nutrition and preparation. In: MARTIN, R. E.; FLINCK, G. J. The seafood industry. New York: Van Nostrand Reinhold, 1990. cap 16, p. 255 - 267.

FRANCO, B. D. G. M.; GUTH, B. E. C. Isolamento e caracterização de cepas de Escherichia coli isoladas de alimentos. Revista de Microbiologia, São Paulo, v. 16, n. 1 p. 49 -55, 1985.

GEROMEL, E. J.; FORSTER, R. J. Princípios fundamentais em tecnologia de pescados. São Paulo: Governo do Estado de São Paulo, Secretaria da indústria, Comércio, Ciência e Tecnologia e Coordenadoria da indústria e comércio, 1982.

KANNER, J. Oxidative process in meat and meat products - Quality implications. Meat Science, Barking, v. 36, n. 1-2, p. 169-189, feb., 1994. Special issue.

KHAYAT, A.; SCHWALL, D. Lipid oxidation in seafood. Food Technology, Chicago, v. 37, n. 7, p. 130-140, 1983.

LOURENÇO, L. F. H.; FERNANDES, G. M. L.; CINTRA, I. H. A. Características físicas, químicas e microbiológicas do mapará (Hypophthalmus edentatus, Spix 1829) salgado e seco em secador solar. In: CONGRESSO BRASILERIO DE ENGENHARIA DE
PESCA; CONGRESSO LATINO-AMERICANODE ENGENHARIA DE PESCA, 11; 1, 1999, Pernambuco. Anais... Recife, 1999, p. 17-21.

BRASIL. Agência Nacional de Vigilância Sanitéria - ANVISA. Portaria n. 451, de 19 de setembro de 1997. Aprova o Regulamento Técnico Princípios Gerais para o Estabelecimento de Critérios e Padrões Microbiológicos para Alimentos e seus Anexos I, II e III. D.O.U. Diário Oficial da União; Poder Executivo, Brasília. 22 set. 1997.

NETO, A. C.; SILVA, C. G. M.; STAMFORD, T. L. Staphylococcus enterotoxigênicos em alimentos in natura e processados no estado de Pernambuco, Brasil. Ciência e Tecnologia de Alimentos, Campinas, v. 22, n. 3, p. 263-271, 2002.

OLIVEIRA, P. R.; SOARES, R. C. Avaliação da composição química e o ponto de congelamento do jaraqui (Semaprochilodus ssp) e mapará (Hypophthalmus ssp). In: CONGRESSO BRASILERIO DE ENGENHARIA DE PESCA;CONGRESSO LATINO-AMERICANO DE ENGENHARIA DE PESCA, 11: 1, 1999, Pernambuco. Anais... Recife, 1999. p. 346-353.

PEREIRA, M. L. et al. Enterotoxigenic Staphylococci from food handlers working in an industrial kitchen in Belo Horizonte, MG (Brazil). Revista de Microbiologia, São Paulo, v. 25, n. 3, p. 161-165, 1994.

RAOULT- WACK, A. L. et al. Osmotic dehydration of mass transfer in terms of engineering properties. In: MUJUMDAR, A. S.; ROQUES, M. A. Drying of solids. New York: Hemisphere Publishing Company, 1989. p. 487-495.

RAOULT- WACK, A. L. Recent advances in the osmotic dehydration of food. Trends in Food Science \& Technology, Cambridge, v. 5, n. 8, p. 225-260, 1994.

ROITMAN, I.; TRAVASSOS, L. R.; AZEVEDO, J. L. Tratado de Microbiologia. São Paulo: Manole, 1988. v. 1, 186 p.

TARLADGIS, B. G. et al. A distillation method for the quantitative determination of malonaldehyde in rancid foods. Journal of American Oil Chemists Society, Chicago, v. 37, n. 1, p. 44-48, jan., 1960.

TORREGGIANI, D. Osmotic dehydration in fruit and vegetables processing. Food Research International, Monticello, v. 26, n. 1, p. 59-68, 1993. 